Supporting Information

\title{
Wearable Porous Au Smartsensors for On-site Detection of Multiple Metal lons
}

\author{
Yu Jiang ${ }^{+}$, Liuxue Shen ${ }^{+}$, Junlin $\mathrm{Ma}^{+}$, Hongting $\mathrm{Ma}^{+}, \mathrm{Yan} \mathrm{Su}^{\ddagger}$ and $\mathrm{Nan} \mathrm{Zhu}^{+}, *$ \\ †Zhang Dayu School of Chemistry, Dalian University of Technology, Dalian, Liaoning, 116024, China. \\ ${ }^{\ddagger}$ School of Chemical Engineering, Dalian University of Technology, Dalian, Liaoning, 116024, China \\ AUTHOR INFORMATION \\ Corresponding Author \\ *E-mail: nanzhu@dlut.edu.cn. \\ ORCID
}

Nan Zhu: 0000-0001-6029-6200 


\section{Table of contents}

Figure S1. Standardization of $\mathrm{Ag} / \mathrm{AgCl}$ ink-based reference electrode.

Figure S2. Optical image of flexible sensor.

Figure S3. TEM of (a) PB-AuNPs and (b) Porous Au.

Figure S4. XPS spectrum.

Figure S5. Comparison between XRD spectrum of PB-AuNPs and porous Au.

Figure S6. CV of electrodeposition process by electroplating solution of $\mathrm{PB}$ without $\mathrm{HAuCl}_{4}$.

Figure S7. CV of co-electrodeposition and activated process.

Figure S8. Electrochemical impedance spectroscopy (EIS).

Figure S9. Calibration curves of $\mathrm{Pb}, \mathrm{Cu}$, and $\mathrm{Hg}$ under different concentration.

Figure S10. Anti-interference study.

Figure S11. Repeatability of heavy metal sensors.

Figure S12. Calibration curves of real-time monitoring for three metal ions by a smartphone.

Figure S13. Wearable sensors for actual samples detection.

Table S1. Comparison of various traditional electrochemical sensors and this porous Au-based wearable sensor for heavy metal ions analysis.

\section{Reference}



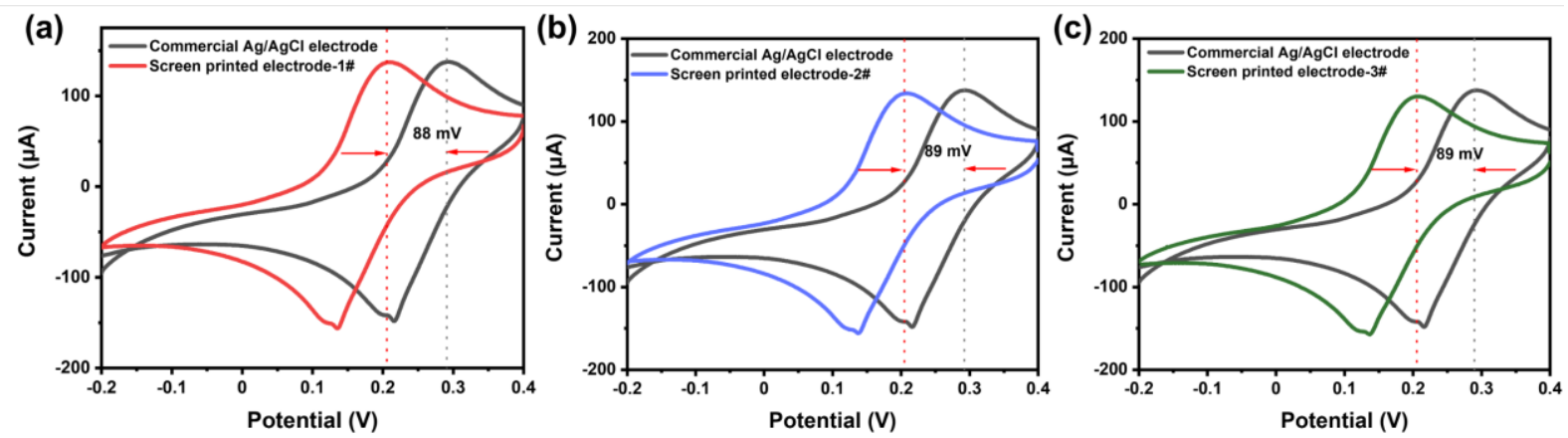

Figure S1. Comparison of three different wearable sensing arrays by screen printing and commercial $\mathrm{Ag} / \mathrm{AgCl}$ electrode as reference electrode by $\mathrm{CV}$ curves. Electrolyte was $5 \mathrm{mM} \mathrm{K} \mathrm{K}_{3} \mathrm{Fe}(\mathrm{CN})_{6}$ containing $1 \mathrm{M} \mathrm{KCl}$. 
(a)

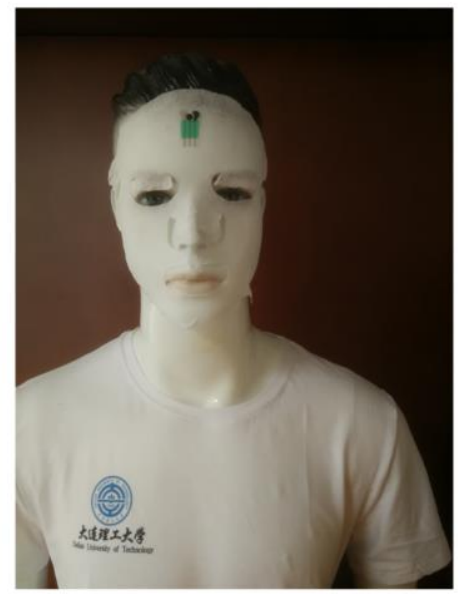

(b)

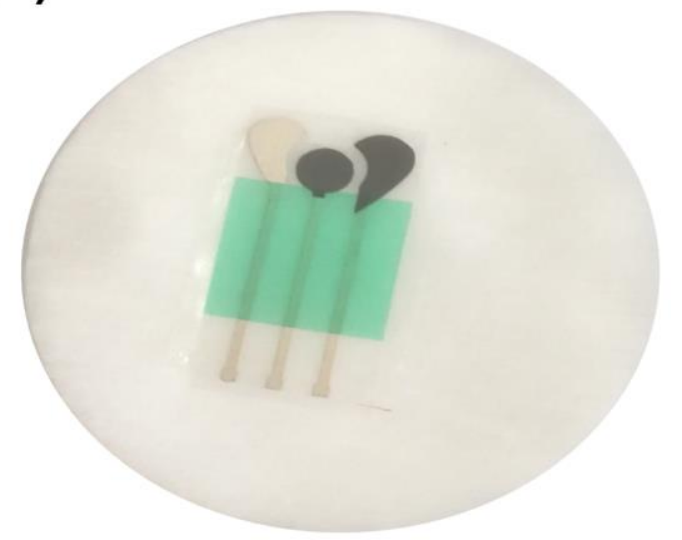

Figure S2. Optical image of flexible sensor modified on (a) facial mask tissue and (b) makeup cotton. 

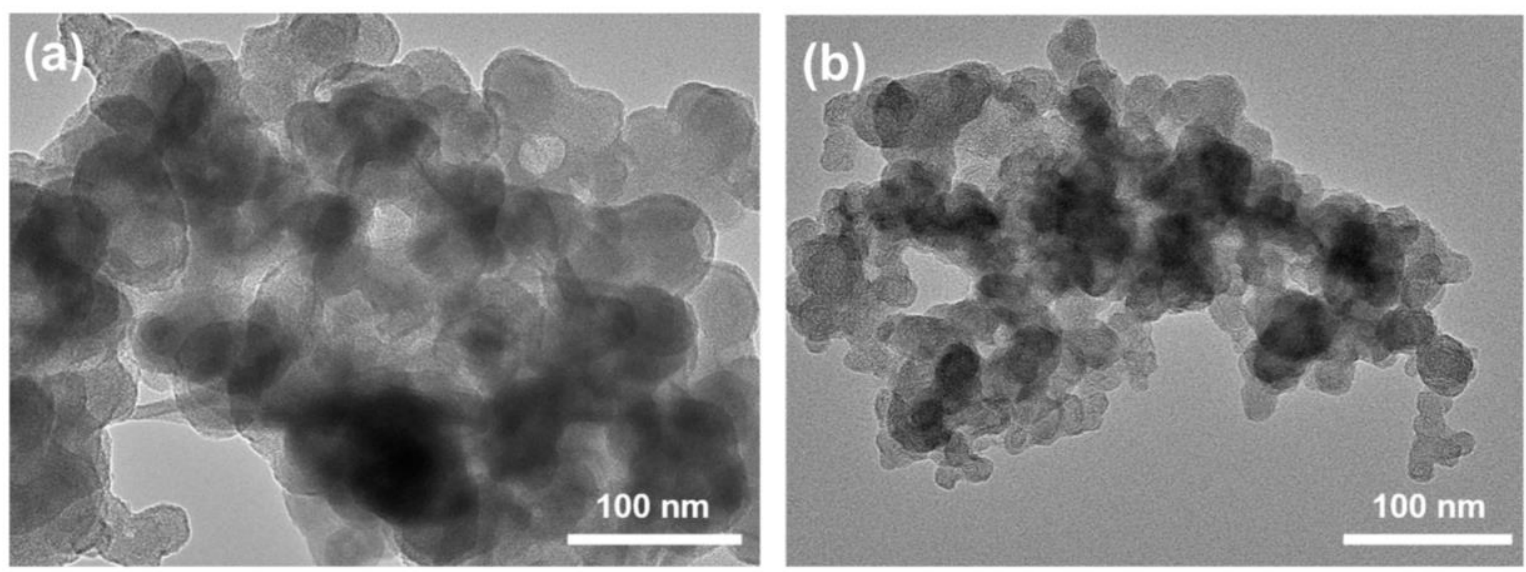

Figure S3. TEM of (a) PB-AuNPs and (b) Porous Au. 

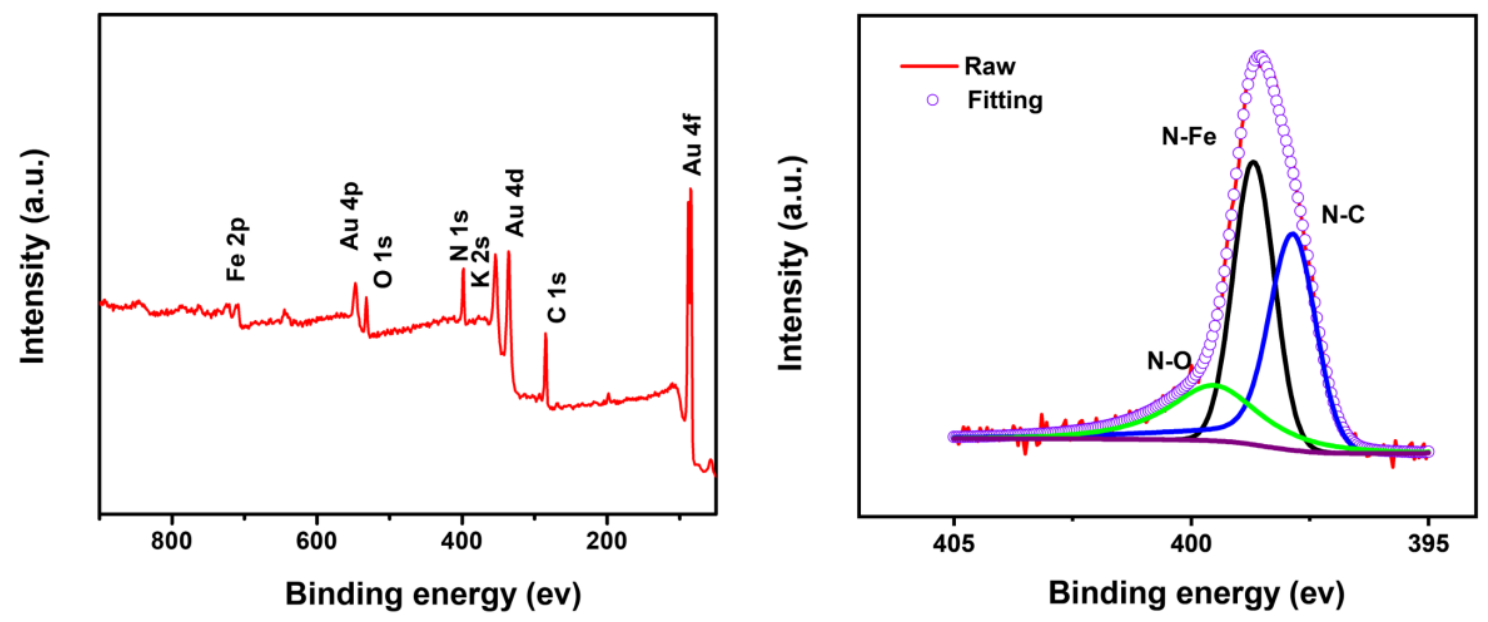

Figure S4. (a) Wide-scan XPS spectrum. (b) High-resolution N 1s XPS spectrum of PB-AuNPs. 


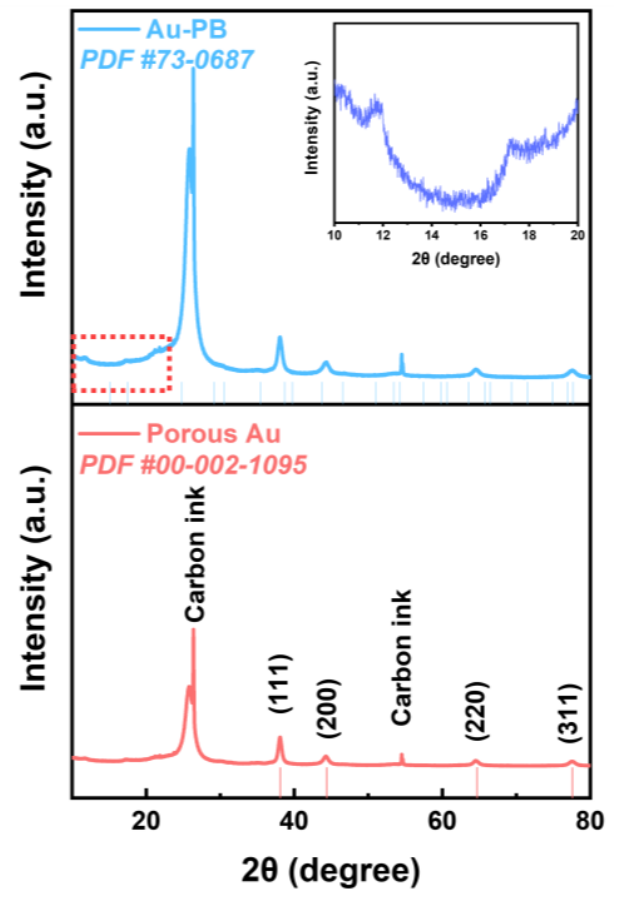

Figure S5. Comparison of XRD spectrum between PB-AuNPs and porous Au.

XRD of PB-AuNPs and porous Au were shown in Figure S5. Compared with PB-AuNPs structure, diffraction peaks at $2 \theta$ among $30-80^{\circ}$ of porous Au had same positions of PB-AuNPs but with sharper intensities, resulting from highly dispersed PB template in PB-AuNPs sample. As depicted in "Preparation and Formation Mechanism of Wearable Porous Au-based Sensors", PB would be simultaneously formed with Au nucleation growing, equally to "co-electrodeposition". Thus, PB was the framework, and Au was well dispersedly loaded in PB skeleton. Due to highly dispersed Au, diffraction peaks of $\mathrm{PB}$ were easily covered by strong diffraction peaks of $\mathrm{Au}$, being difficult to be observed in XRD of PB-AuNPs. Typical diffraction peak of $\mathrm{PB}$ at $2 \theta$ in range of $10-20^{\circ}$ can be observed in magnified views (Figure S5), demonstrating structure of PB in mixture of PB-AuNPs. 


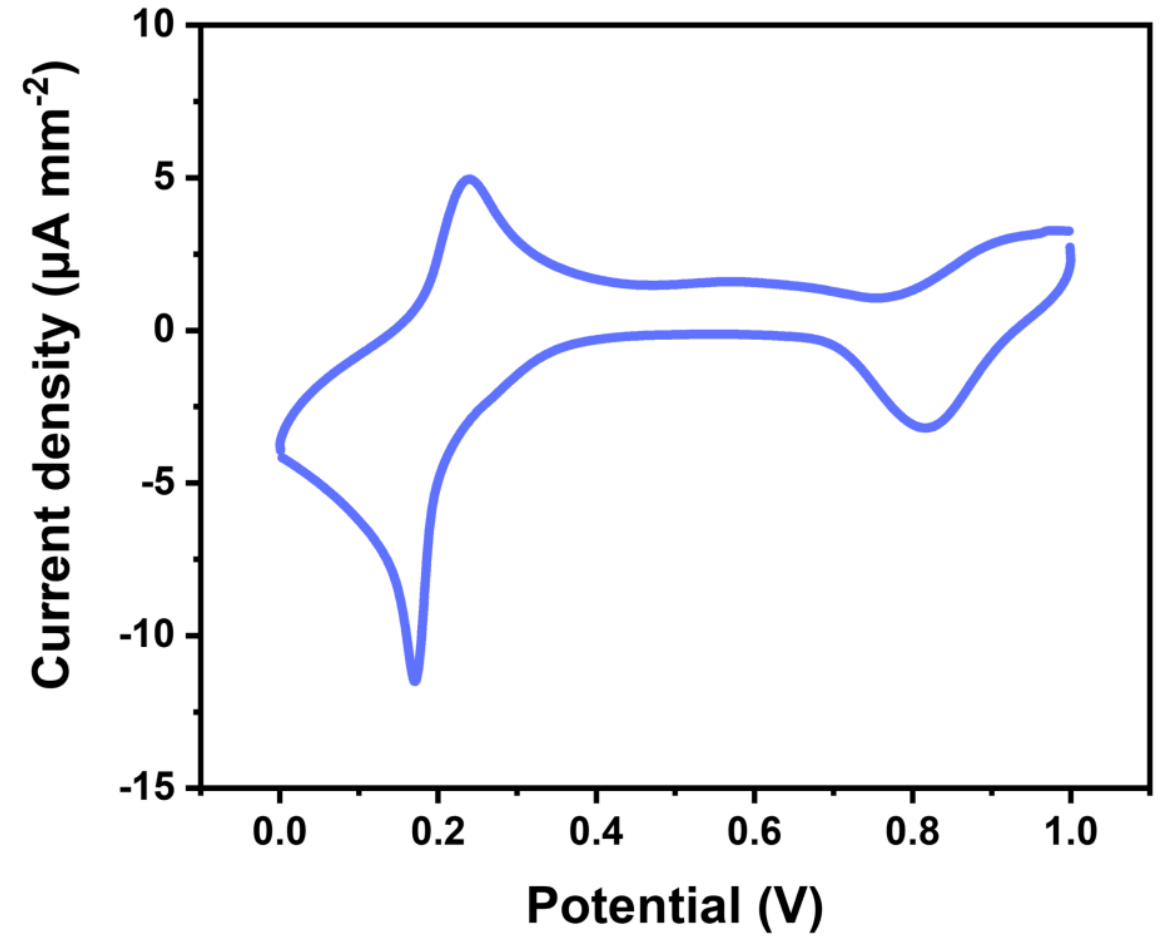

Figure S6. CV of electrodeposition process by electroplating solution of $\mathrm{PB}$ without $\mathrm{HAuCl}_{4}$. Scan rate: $0.05 \mathrm{~V} \mathrm{~s}^{-1}$. 

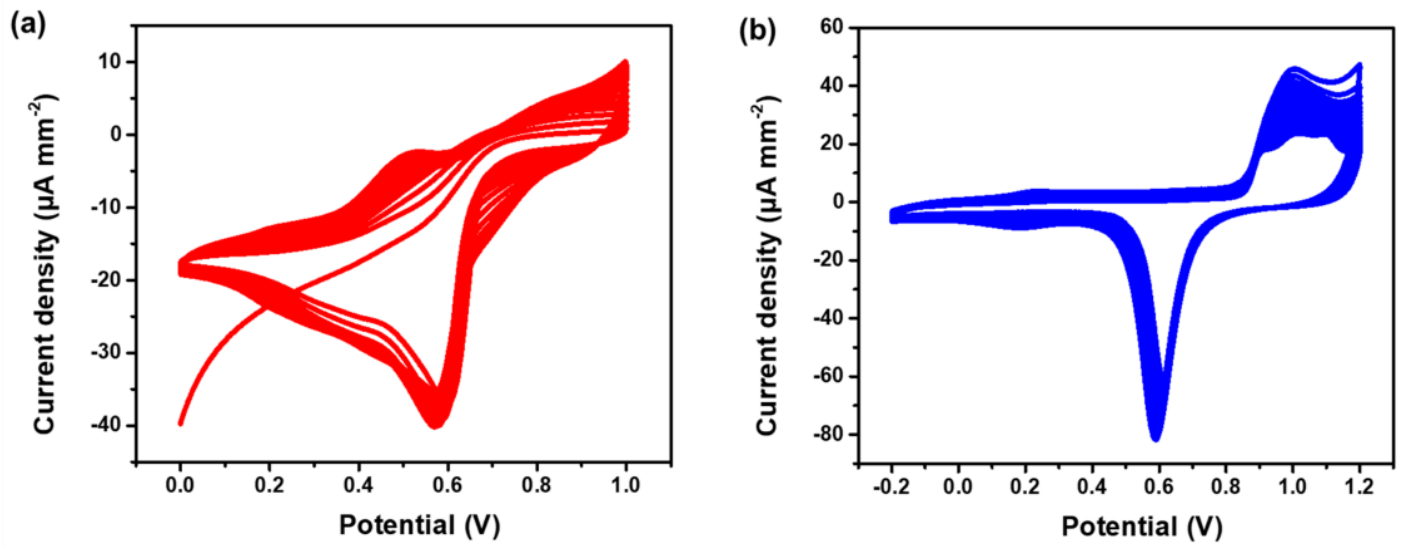

Figure S7. (a) CV of co-electrodeposition process of PB-AuNPs, 60 cycles. Scan rate: $0.05 \mathrm{~V} \mathrm{~s}^{-1}$. (b) Activated process of porous Au sensors, 60 cycle. Scan rate: $0.1 \mathrm{~V} \mathrm{~s}^{-1}$. 


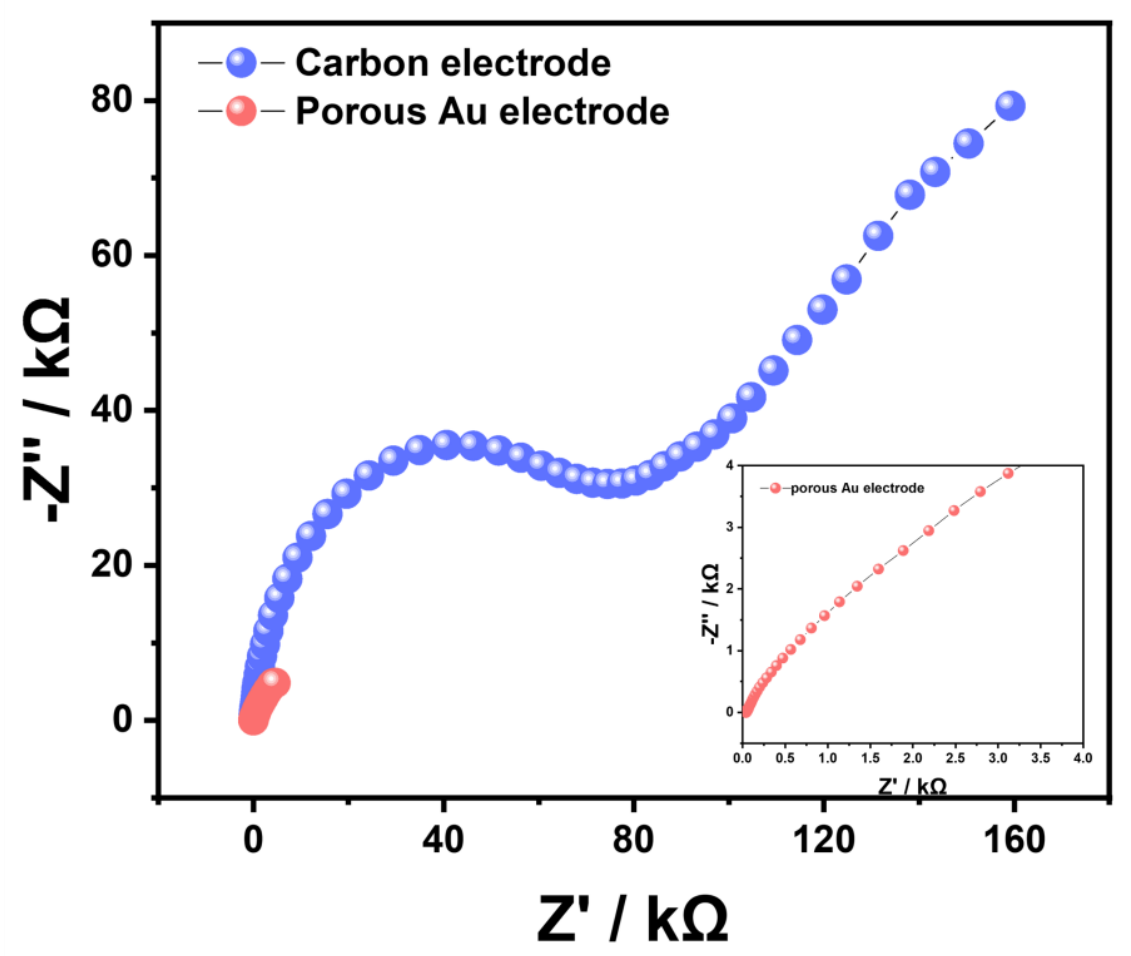

Figure S8. Electrochemical impedance spectroscopy (EIS) plots of carbon-based sensors and porous Au-based sensors at a fixed potential of $0.29 \mathrm{~V}(\mathrm{vs} \mathrm{Ag} / \mathrm{AgCl})$ with frequency range of 100000 to 0.01 $\mathrm{Hz}$. Electrolyte was $5 \mathrm{mM} \mathrm{K}{ }_{3} \mathrm{Fe}(\mathrm{CN})_{6}$ containing $1 \mathrm{M} \mathrm{KCl}$ with an amplitude of $5 \mathrm{mV}$, and inset was magnified views of porous $\mathrm{Au}$ sensor. 

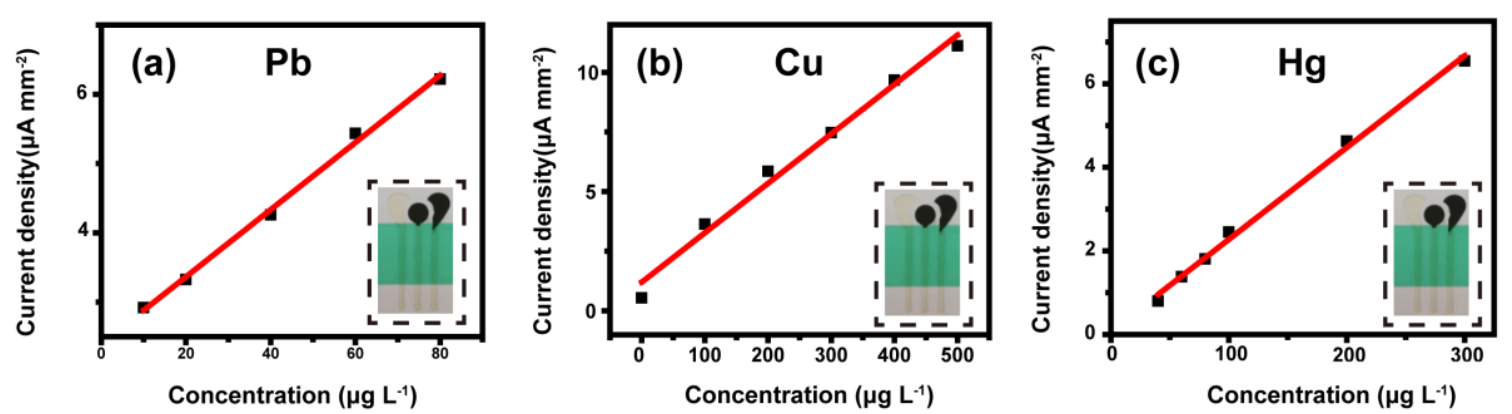

Figure S9. Calibration curves of (a) $\mathrm{Pb},(\mathrm{b}) \mathrm{Cu}$, and (c) Hg under different concentration, respectively (porous $\mathrm{Au}$ as the working electrode, carbon ink as the counter electrode and silver ink as the reference electrode). 

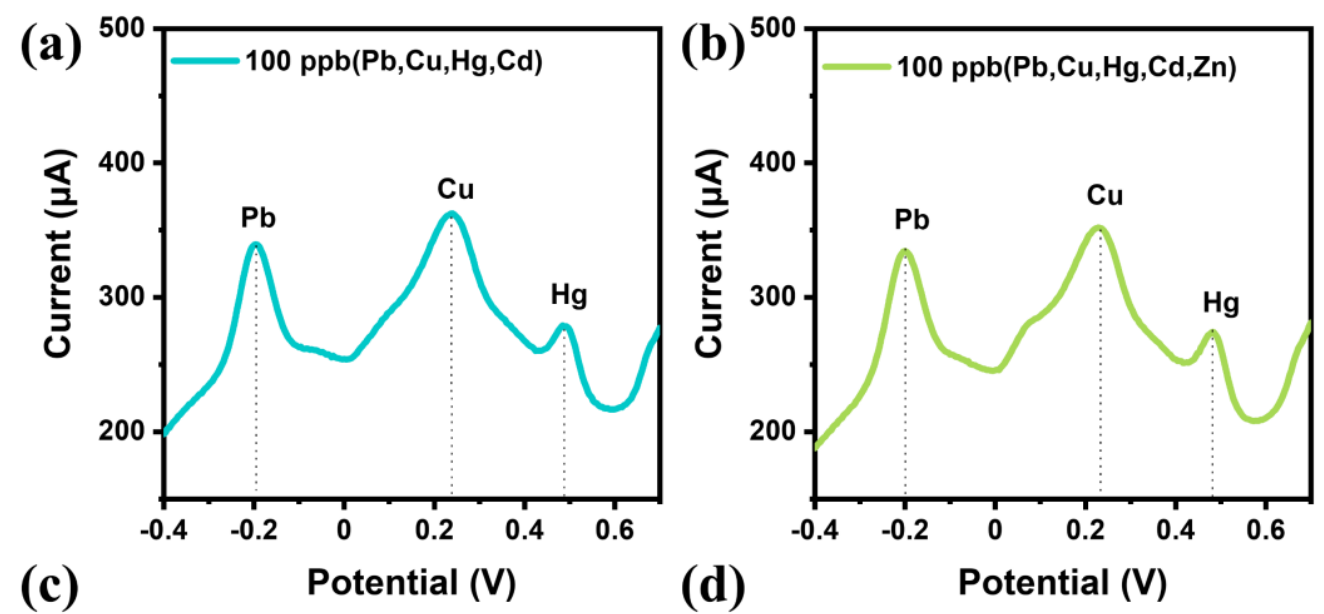

(c)

Potential (V)
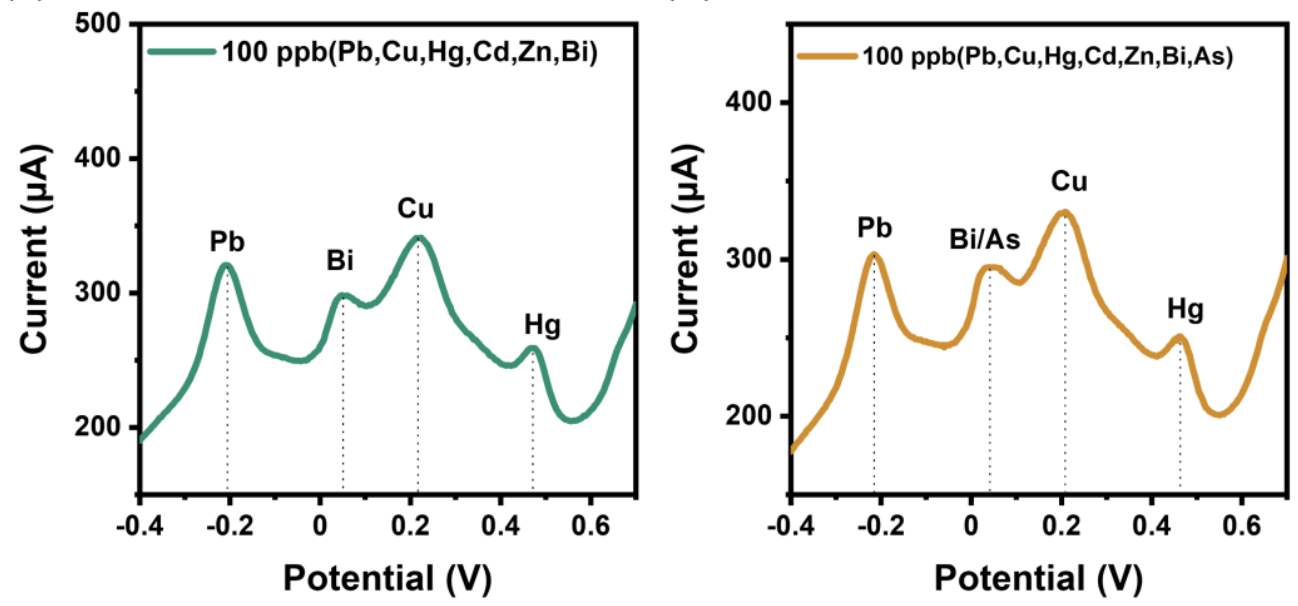

Figure S10. Anti-interference study of porous Au-based smartsensor. Stripping voltammograms under acetate buffer solution with adding (a) $100 \mathrm{ppb} \mathrm{Pb}^{2+}+100 \mathrm{ppb} \mathrm{Cu}^{2+}+100 \mathrm{ppb} \mathrm{Hg}^{2+}+100 \mathrm{ppb} \mathrm{Cd}^{2+}$, (b)

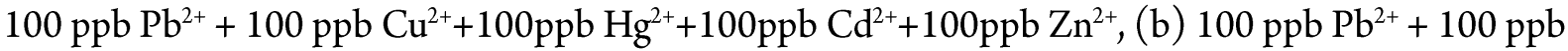

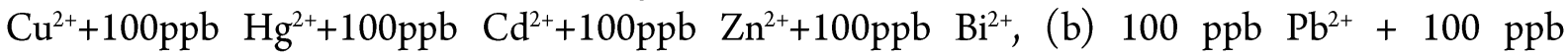
$\mathrm{Cu}^{2+}+100 \mathrm{ppb} \mathrm{Hg}{ }^{2+}+100 \mathrm{ppb} \mathrm{Cd}^{2+}+100 \mathrm{ppb} \mathrm{Zn}^{2+}+100 \mathrm{ppb} \mathrm{Bi}^{2+}+100 \mathrm{ppb} \mathrm{As}^{3+}$. 

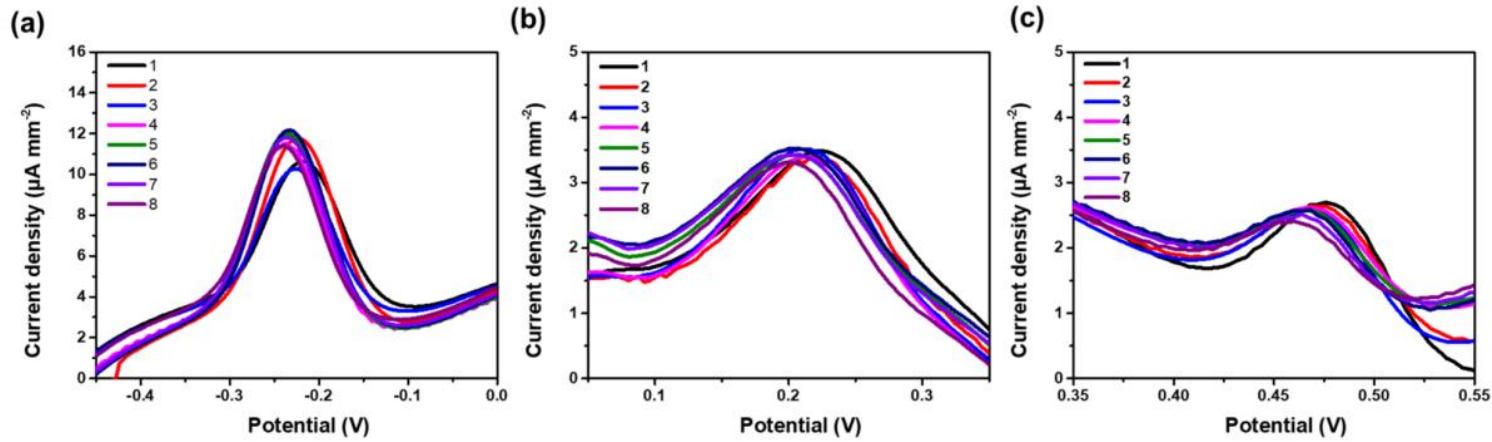

Figure S11. Repeatability of heavy metal sensors for detection of (a) $\mathrm{Pb},(\mathrm{b}) \mathrm{Cu},(\mathrm{c}) \mathrm{Hg}$ under 100 ppb. 

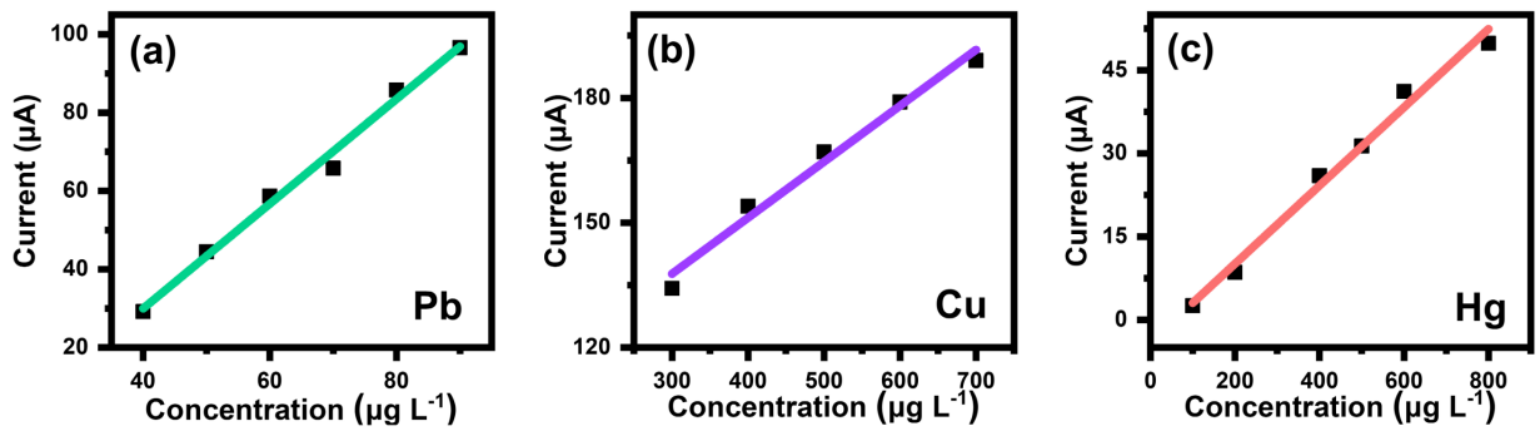

Figure S12. Calibration curves of real-time monitoring for three heavy metal ions by a smartphone. 

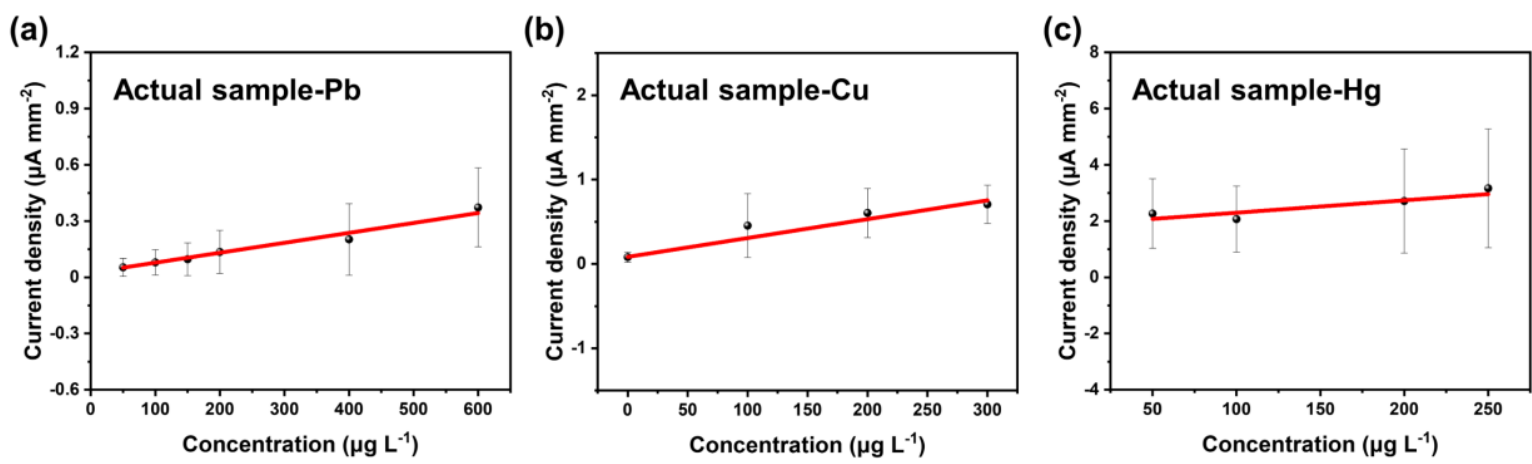

Figure S13. Wearable sensors for actual samples detection. Corresponding linear relationship plots in detection of (a) Pb, (b) Cu and (c) Hg. 
Table S1. Comparison of various traditional electrochemical sensors with porous Au-based wearable sensor for heavy metal ions analysis.

\begin{tabular}{|c|c|c|c|c|c|c|}
\hline $\begin{array}{c}\text { Metal } \\
\text { ions }\end{array}$ & Electrode materials & $\begin{array}{l}\text { Detection } \\
\text { technology }\end{array}$ & Limit of detection (LOD) & Sensitivity & Stability & Reference \\
\hline $\begin{array}{l}\mathrm{Cu}^{2+} \\
\mathrm{Pb}^{2+} \\
\mathrm{Hg}^{2+}\end{array}$ & $\begin{array}{l}\text { EDTA-PANI/SWCNTs } \\
\text { nanocomposite }\end{array}$ & DPV & $\begin{array}{c}\mathrm{Cu}^{2+}: 0.08 \mu \mathrm{M} \\
\mathrm{Pb}^{2+}: 1.65 \mu \mathrm{M}, \mathrm{Hg}^{2+}: 0.68 \mu \mathrm{M}\end{array}$ & - & - & {$[1]$} \\
\hline $\mathrm{Cu}^{2+}$ & $\begin{array}{l}\text { EDTA-modified } \\
\text { PANI/SWNTs } \\
\text { nanocomposite }\end{array}$ & DPV & $1.4 \mu \mathrm{M}$ & $189 \mathrm{mAM}^{-1}$ & - & [2] \\
\hline $\mathrm{Cu}^{2+}$ & PANI/ITO-electrode & $\begin{array}{l}\text { electrochemical/el } \\
\text { ectrochromic }\end{array}$ & - & - & - & {$[3,4]$} \\
\hline $\begin{array}{l}\mathrm{Cd}^{2+} \\
\mathrm{Pb}^{2+} \\
\mathrm{Cu}^{2+} \\
\mathrm{Hg}^{2+}\end{array}$ & $\begin{array}{c}\text { Graphene } \\
\text { Aerogel-Metal-Organic } \\
\text { Framework composites }\end{array}$ & DPSV & $\begin{array}{c}\mathrm{Cd}^{2+}: 0.02 \mu \mathrm{M}, \\
\mathrm{Pb}^{2+}: 1.5 \mathrm{nM}, \\
\mathrm{Cu}^{2+}: 7 \mathrm{nM}, \\
\mathrm{Hg}^{2+}: 2 \mathrm{nM}\end{array}$ & $\begin{array}{c}\mathrm{Cd}^{2+}: 0.4953 \mu \mathrm{A} \\
\mu \mathrm{M}^{-1}, \\
\mathrm{~Pb}^{2+}: 0.651 \mathrm{Ma} \\
\mu \mathrm{M}^{-1}, \\
\mathrm{Cu}^{2+}: 0.7314 \mu \mathrm{A} \\
\mu \mathrm{M}^{-1}, \\
\mathrm{Hg}^{2+}: 0.5392 \mu \mathrm{A} \\
\mu \mathrm{M}^{-1}\end{array}$ & - & {$[5]$} \\
\hline $\mathrm{Pb}^{2+}$ & Bismuth-based carbon ink & SWASV & $4.8 \mathrm{nM}$ & $0.17 \mu \mathrm{A} \mathrm{L} \mu \mathrm{g}^{-1}$ & - & [6] \\
\hline $\begin{array}{l}\mathrm{Cd}^{2+} \\
\mathrm{Pb}^{2+}\end{array}$ & $\begin{array}{l}\text { phytic acid functionalized } \\
\text { polypyrrole/graphene } \\
\text { oxide nanocomposites }\end{array}$ & DPV & $\begin{array}{l}\mathrm{Cd}^{2+}: 0.02 \mu \mathrm{M} \\
\mathrm{Pb}^{2+}: 1.97 \mathrm{nM}\end{array}$ & $\begin{array}{c}\mathrm{Cd}^{2+}: 0.18 \mu \mathrm{AL} \\
\mu \mathrm{g}^{-1} \\
\mathrm{~Pb}^{2+}: 0.27 \mu \mathrm{AL} \\
\mu \mathrm{g}^{-1}\end{array}$ & - & [7] \\
\hline $\begin{array}{l}\mathrm{Pb}^{2+} \\
\mathrm{Cu}^{2+} \\
\mathrm{Hg}^{2+}\end{array}$ & $\begin{array}{l}\text { Porous Au-based screen- } \\
\text { printed electrode }\end{array}$ & SWV & $\begin{array}{l}\mathrm{Pb}^{2+}: 0.03 \mu \mathrm{M} \\
\mathrm{Cu}^{2+}: 0.85 \mu \mathrm{M} \\
\mathrm{Hg}^{2+}: 0.07 \mu \mathrm{M}\end{array}$ & $\begin{array}{c}\mathrm{Pb}^{2+}: 0.048 \mu \mathrm{AL} \\
\mu \mathrm{g}^{-1} \mathrm{~mm}^{-2}, \\
\mathrm{Cu}^{2+}: 0.021 \mu \mathrm{AL} \\
\mu \mathrm{g}^{-1} \mathrm{~mm}^{-2}, \\
\mathrm{Hg}^{2+}: 0.022 \mu \mathrm{AL} \\
\mu \mathrm{g}^{-1} \mathrm{~mm}^{-2}\end{array}$ & One week & This work \\
\hline
\end{tabular}

\section{Reference}

(1) Deshmukh, M. A.; Celiesiute, R.; Ramanaviciene, A.; Shirsat, M. D.; Ramanavicius, A.,EDTA_PANI/SWCNTs nanocomposite modified electrode for electrochemical determination of copper (II), lead (II) and mercury (II) ions, Electrochim. Acta 2018, 259, 930-938.

(2) Deshmukh, M. A.; Patil, H. K.; Bodkhe, G. A.; Yasuzawa, M.; Koinkar, P.; Ramanaviciene, A.; Shirsat, M. D.; Ramanavicius, A.,EDTAmodified PANI/SWNTs nanocomposite for differential pulse voltammetry based determination of $\mathrm{Cu}$ (II) ions, Sens. Actuators, B 2018, 260, 331-338.

(3) Deshmukh, M. A.; Shirsat, M. D.; Ramanaviciene, A.; Ramanavicius, A.,Composites Based on Conducting Polymers and Carbon Nanomaterials for Heavy Metal Ion Sensing (Review), Crit. Rev. Anal. Chem. 2018, 48 (4), 293-304.

(4) Deshmukh, M. A.; Gicevicius, M.; Ramanaviciene, A.; Shirsat, M. D.; Viter, R.; Ramanavicius, A.,Hybrid electrochemical/electrochromic $\mathrm{Cu}$ (II) ion sensor prototype based on PANI/ITO-electrode, Sens. Actuators, B 2017, 248, 527-535.

(5) Lu, M.; Deng, Y.; Luo, Y.; Lv, J.; Li, T.; Xu, J.; Chen, S. W.; Wang, J.,Graphene Aerogel-Metal-Organic Framework-Based Electrochemical Method for Simultaneous Detection of Multiple Heavy-Metal Ions, Anal. Chem. 2019, 91 (1), 888-895.

(6) Nie, Z.; Nijhuis, C. A.; Gong, J.; Chen, X.; Kumachev, A.; Martinez, A. W.; Narovlyansky, M.; Whitesides, G. M.Electrochemical sensing in paper-based microfluidic devices, Lab Chip 2010, 10 (4), 477-483.

(7) Dai, H.; Wang, N.; Wang, D.; Ma, H.; Lin, M.,An electrochemical sensor based on phytic acid functionalized polypyrrole/graphene oxide nanocomposites for simultaneous determination of $\mathrm{Cd}$ (II) and $\mathrm{Pb}(\mathrm{II})$, Chem. Eng. J. 2016, 299, 150-155. 